\title{
Optical Gain in Ultrathin Self-Assembled Bi-Layers of Colloidal Quantum Wells Enabled by the Mode Confinement in their High-Index Dielectric Waveguides
}

\author{
Sina Foroutan-Barenji, Onur Erdem, Negar Gheshlaghi, Yemliha Altintas, \\ and Hilmi Volkan Demir*
}

This study demonstrates an ultra-thin colloidal gain medium consisting of bi-layers of colloidal quantum wells (CQWs) with a total film thickness of $14 \mathrm{~nm}$ integrated with high-index dielectrics. To achieve optical gain from such an ultra-thin nanocrystal film, hybrid waveguide structures partly composed of self-assembled layers of CQWs and partly high-index dielectric material are developed and shown: in asymmetric waveguide architecture employing one thin film of dielectric underneath CQWs and in the case of quasi-symmetric waveguide with a pair of dielectric films sandwiching CQWs. Numerical modeling indicates that the modal confinement factor of ultra-thin CQW films is enhanced in the presence of the adjacent dielectric layers significantly. The active slabs of these CQW monolayers in the proposed waveguide structure are constructed with great care to obtain near-unity surface coverage, which increases the density of active particles, and to reduce the surface roughness to sub-nm scale, which decreases the scattering losses. The excitation and propagation of amplified spontaneous emission (ASE) along these active waveguides are experimentally demonstrated and numerically analyzed.

The findings of this work offer possibilities for the realization of ultra-thin electrically driven colloidal laser devices, providing critical advantages including single-mode lasing and high electrical conduction.

\section{Introduction}

Optical gain and lasing from solutionprocessable materials have attracted great interest for a wide range of material systems including organic semiconductors, ${ }^{[1]}$ perovskites, ${ }^{[2]}$ and semiconductor nanocrystals. ${ }^{[3-5]}$ These classes of soft materials simplify the fabrication process, and as a result, their device fabrication costs can be reduced tremendously. Successful realization of solution-processed lasing devices could expand the use of lasing technology and open up exciting low-cost applications including those in chemical sensing, ${ }^{[6]}$ biological imaging, ${ }^{[7]}$ and lab-on-a-chip diagnostics. ${ }^{[8]}$

Among such solution-processable materials, colloidal quantum wells (CQWs), the atomically flat member of semiconductor nanocrystal family, have been the focus of increasing attention thanks to their excellent electronic and optical properties for optoelectronic applications. ${ }^{\left[{ }^{9-12}\right]}$ CQWs

S. Foroutan-Barenji, Dr. O. Erdem, Dr. N. Gheshlaghi, Dr. Y. Altintas, Prof. H. V. Demir

Department of Electrical and Electronics Engineering

Department of Physics

UNAM - Institute of Materials Science and Nanotechnology

Bilkent University

Ankara 06800, Turkey

E-mail:volkan@bilkent.edu.tr, hvdemir@ntu.edu.sg

Dr. Y. Altintas

Department of Materials Science and Nanotechnology

Abdullah Gül University

Kayseri TR-38080, Turkey

Prof. H. V. Demir

LUMINOUS! Centre of Excellence for Semiconductor Lighting and Displays

Centre of Optical Fiber Technology

The Photonics Institute

School of Electrical and Electronic Engineering

School of Physical and Mathematical Sciences

Nanyang Technological University

50 Nanyang Avenue, Singapore 639798, Singapore

The ORCID identification number(s) for the author(s) of this article can be found under https://doi.org/10.1002/smll.202004304.

DOI: 10.1002/smll.202004304 exhibit narrow spontaneous emission spectra, suppressed inhomogeneous broadening, giant oscillator strength, and high density of energy states at the band edge, which are highly desired for optical gain and lasing. ${ }^{[13-15]}$

Optical gain and lasing using a few hundred nanometers to micrometer thick CQW active regions have thus far been reported..$^{5,16]}$ However, achieving gain from ultra-thin active CQW film has been a major challenge. The thinnest CQW gain medium reported so far consists of six layers of CQWs, which corresponds to a film thickness of $42 \mathrm{~nm} \cdot{ }^{[17]}$ Realizing further thinner colloidal gain media has remained impossible to date.

The realization of electrically driven colloidal lasers is similarly challenging, since the thick layers of colloidal nanocrystals nominally required for gain, which are typically capped with long organic molecules, suffer from low electrical conductivity. To overcome the issue of electrical conduction, the original bulky ligands used for surface passivation can be replaced with shorter ligands, but this does not work in favor of the device performance as this process results in an increase of defect or trap states in the ligandexchanged particles leading to nonradiative losses, which in turn reduces the gain performance. ${ }^{[18,19]}$ Hence, one way of increasing the conduction is to reduce the thickness of gain film. 
However, a big hindrance for making gain medium thinner is Auger recombination, which competes with optical gain. Thinner active films suffer from lower optical mode confinement, hence the modal gain is not able to overcome losses. Therefore, one needs to pump harder to make up for the reduced modal gain, but then Auger process kicks in. To suppress Auger loss mechanism, various material engineering strategies including growing giant alloyed shells have been introduced to reduce the core/shell lattice mismatch. ${ }^{[9]}$ Although the methods used for material engineering have been successful in reducing loss mechanisms to some extent, the required active film thickness is still typically larger than $100 \mathrm{~nm}$ in these giant nanocrystals..$^{[9,15,20]}$

To address the gain performance issue of thin films, we also need to consider the film preparation methods, since the film quality can help reduce losses and improve the gain performance significantly. Spin-coating and drop-casting have been the common techniques for preparing thin films of colloidal nanocrystals. ${ }^{[16,20,21]}$ The films prepared by these methods are not sufficiently dense or uniform. Also, these methods do not give high precision in thickness control of CQW films and yield random orientation of CQWs in film, which introduces surface roughness and increases scattering losses. To solve this problem, our group has recently reported a new self-assembly method, which allows for orientationcontrolled deposition of CQWs monolayers on various substrates with near-unity surface coverage. ${ }^{[22]}$ Importantly, CQW films in the face-down configuration has shown dipole orientation within the plane of the films, which can enhance the external efficiency beyond what can be achieved with isotropic emitters. ${ }^{[23]}$ Furthermore, this method reduces the CQW consumption during the device fabrication by a factor of about 10 in comparison to spin-coating for the same thickness of gain media. This technique provides us with an opportunity for the development of low-cost device production for commercial applications and mass production.

The approach for monolayer deposition of CQWs was also further extended with some modifications to obtain multilayered CQW constructs with precisely tunable film thickness and sub-nm surface roughness. ${ }^{[17]}$ In this previous work, by depositing such multiple layers of CQWs on a bare fused silica substrate, the minimum thickness at which optical gain has been observed was 6 monolayers of CQWs, corresponding to about $42 \mathrm{~nm}$, below which there was no supported optical mode observed. To achieve optical gain at further lower film thicknesses, additional means to induce gain are therefore needed.

Implementing cavities and waveguides into quantum dots (QDs) have been investigated and efficient coherent light sources have been achieved.24-26] However, achieving optical gain from an ultra-thin colloidal medium using optical structures remains a challenge to date. Integrating an extremely thin CQW gain medium into a carefully designed optical structure with an optimum optical mode confinement represents a promising methodology for achieving gain from an ultra-thin few monolayers of CQWs. Furthermore, this type of ultra-thin gain structure can enable a variety of applications in different domains including on-chip downscaling of photonic integrated devices and enabling the studies of extreme interactions between matter and light such as plasmon-photon coupling. ${ }^{[27]}$
Here, we successfully demonstrate optical gain induced in an ultra-thin CQW bi-layer, in which the optical field is confined within the active layer with the help of stratified high index dielectric media in its vicinity. For this purpose, we propose and demonstrate hybrid waveguides comprising of CdSe/ CdZnS core/shell CQW layers deposited layer-by-layer on top of $\mathrm{TiO}_{2}$ film (asymmetric waveguide). Carefully engineering the refractive index profile of our multilayered waveguide enabled us to attain sufficient overlap between the optical mode and the CQW layer to induce gain from a minimum of three layers of CQWs. Further adopting quasi-symmetric waveguide architecture, consisting of CQW film sandwiched between two $\mathrm{TiO}_{2}$ layers, allowed for gain from an ultra-thin layer made of merely two layers of CQWs, which corresponds to a thickness of $14 \mathrm{~nm}$. To the best of our knowledge, this result of gain medium thickness achieved using the solution-processed materials that exhibit gain is the lowest reported to date. Here the generation and propagation of light in these hybrid waveguides were systematically assessed, both theoretically and experimentally. In particular, we utilized an experimental methodology that allows for obtaining the losses and net gain coefficients for the waveguide slabs of these CQWs with different thicknesses as a function of the pump fluence. We applied a four-level laser model to analyze the net gain data and to extract the material gain, the effective stimulated emission cross-section, and the pump threshold for the onset of ASE, and the pump saturation fluence.

\section{Results and Discussion}

The gain material used in this work is comprised of CdSe/ $\mathrm{Cd}_{0.25} \mathrm{Zn}_{0.75} \mathrm{~S}$ core/hot-injection shell (HIS) CQWs with type-I band structure, which were synthesized using some modifications according to the synthesis recipe reported in our recent studies..$^{[9,28]}$ Figure 1a shows the photoluminescence (PL) and absorption spectra of these CQWs. Figure $1 \mathrm{~b}$ depicts the highresolution transmission electron microscopy (HR-TEM) image of these CQWs with the average dimensions $(l \times w \times h)$ of $17.6 \pm$ $1.6 \times 16.9 \pm 1.2 \times 4.6 \pm 0.5 \mathrm{~nm}^{3}$. The thickness of these CQWs increases to about $7 \mathrm{~nm}$ if capped oleic acid and oleylamine ligands are taken into account. Recently, Altintas et al. have demonstrated a record-low threshold optical gain and lasing performance from thick films of these HIS CQWs prepared by drop-casting method. ${ }^{[9]}$ However, such thick gain media of CQWs passivated with organic surfactants suffer from low electrical conductivity, heat generation, and scattering losses due to surface roughness as well as multimode formation.

Here we deposited our CQW gain medium in a face-down orientation via our liquid-air interface colloidal self-assembly method. ${ }^{[17]}$ Scanning electron microscopy (SEM) imaging reveals the surface coverage of the self-assembled films to be as high as $90 \%$ (Figure S1, Supporting Information). The calculated effective complex refractive index of this CQW film at $650 \mathrm{~nm}$ wavelength for $90 \%$ surface coverage is $1.96+\mathrm{i} 0.018$ (see the Supporting Information), which is in agreement with the measured complex refractive index of the film using ellipsometry $(1.95+\mathrm{i} 0.020)$, shown in Figure $1 c$. The real part of this refractive index is the effective refractive index for the 
a)

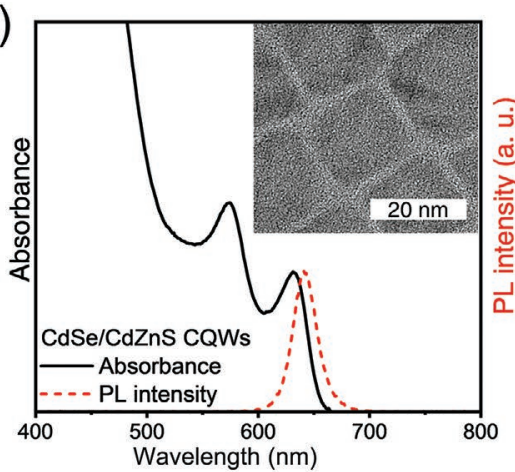

d)

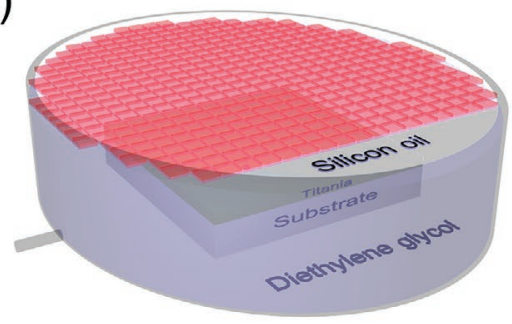

b)

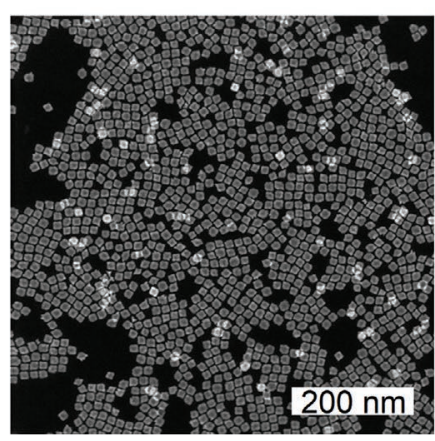

c)

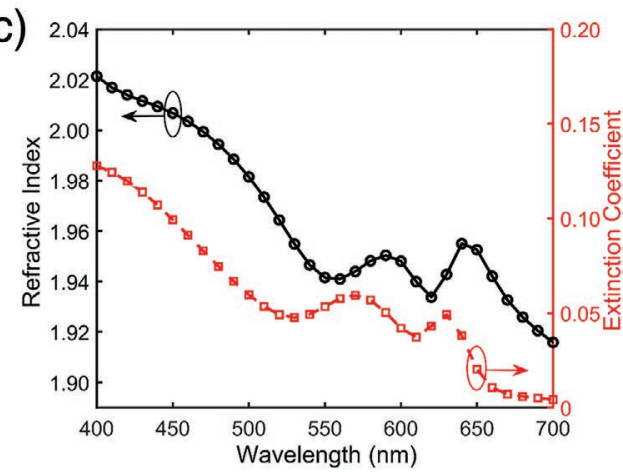

e)

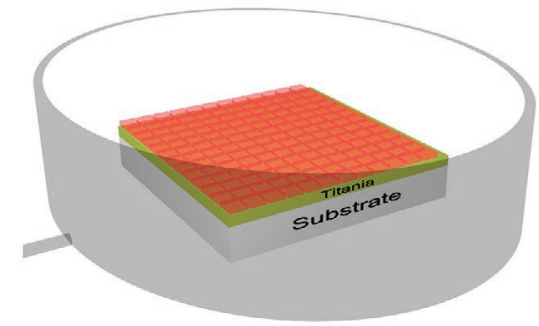

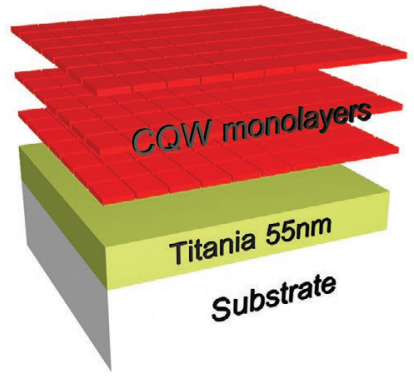

Figure 1. a) PL and absorption spectra of $\mathrm{CdSe} / \mathrm{Cd}_{0.25} \mathrm{Zn}_{0.75} \mathrm{~S}$ core/HIS CQWs. b) HR-TEM image of the CQWs (the scale bar is $200 \mathrm{~nm}$ ). c) Complex refractive index of self-assembled CQW film measured with ellipsometry. d) Liquid-air interface self-assembly procedure. e) Schematic of asymmetric waveguide consisting of a few monolayers of CQWs on top of $\mathrm{TiO}_{2}$ film and fused silica substrate.

optical mode propagating along the CQW plane, across which the CQW dipoles are isotropic. ${ }^{[23,29]}$ On the other hand, ellipsometry measurements on the spin-coated films of the same CQWs yield a refractive index of about 1.80 , due to more sparse packing of CQWs compared to the self-assembly deposited CQWs. Our self-assembly approach therefore forms very uniform, tightly packed CQW film with a negligible amount of voids and sparse imperfections, reducing optical scattering (Figure S2, Supporting Information), and increasing the effective refractive index of the resulting film compared to spincoated films of the same CQWs.

Here the multilayers of these CQWs deposited on bare fused silica display ASE for 7 monolayers (corresponding to $49 \mathrm{~nm}$ thickness) (see Figure S3, Supporting Information). In this study, optical gain with a fewer number of CQW layers was hindered by insufficient optical confinement making it impossible to induce gain from thinner films. To overcome this problem, our hypothesis is that the spatial overlap between the CQW films, self-assembled on top of a high-index material, and the evanescent field of the confined optical mode can be made strong enough to couple the light emitted by CQWs into their films, and thus to enhance the optical confinement with even thinner optically active CQW films.

For this purpose, with a high refractive index of 2.41 at the CQW emission peak (650 nm), $\mathrm{TiO}_{2}$ can be a good candidate (see Figure S4, Supporting Information). The $\mathrm{TiO}_{2}$ films can also help with heat transfer from the CQW active medium, allowing better heat dissipation and stable response due to its higher thermal conductivity compared to using only the fused silica substrate (see Figure S6, Supporting Information). ${ }^{[30]}$
Here, a thin film of $\mathrm{TiO}_{2}$ was deposited on fused silica substrate using atomic layer deposition (ALD), followed by careful deposition of CQW monolayers via our self-assembly method as shown in Figure 1d. We used fused silica as the substrate and diethylene glycol (DEG) as the subphase for the self-assembly of CQW monolayers (see the Experimental Section for more information). The schematics of the resulting asymmetric waveguide consisting of layers of CQWs on a $\mathrm{TiO}_{2}$ thin film is shown in Figure 1e.

Based on the measured refractive index of the self-assembled CQW film (Figure 1c), the optimum thickness of the $\mathrm{TiO}_{2}$ film was systematically studied to obtain the maximum possible confinement factor for different number of CQW layers. Here, the mode confinement factor, $\Gamma$, is defined as the ratio of the mode power inside the CQW film to the total power

$$
\Gamma=\int_{C Q W} \frac{1}{2} \operatorname{Re}\left\{\vec{E} \times \vec{H}^{*}\right\} \cdot \hat{z} d x d y / \int_{-\infty}^{\infty} \frac{1}{2} \operatorname{Re}\left\{\vec{E} \times \vec{H}^{*}\right\} \cdot \hat{z} d x d y
$$

where $\vec{E}$ and $\vec{H}$ are the electric and magnetic fields, respectively. The integral in the numerator is evaluated across the optically active portion of the waveguide, the CQW layers. Figure $2 \mathrm{a}$ shows the fundamental transverse electric $\left(\mathrm{TE}_{0}\right)$ mode confinement factor at the wavelength of interest $(650 \mathrm{~nm})$ as a function of the $\mathrm{TiO}_{2}$ film thickness for different number of CQW layers. For a monolayer of CQWs, the cut-off thickness of $\mathrm{TiO}_{2}$ to support the fundamental mode is about $22 \mathrm{~nm}$. As we increase the $\mathrm{TiO}_{2}$ thickness, the mode confinement also increases and finally reaches a maximum at an optimum thickness, after which the mode confinement reduces. In this case, 


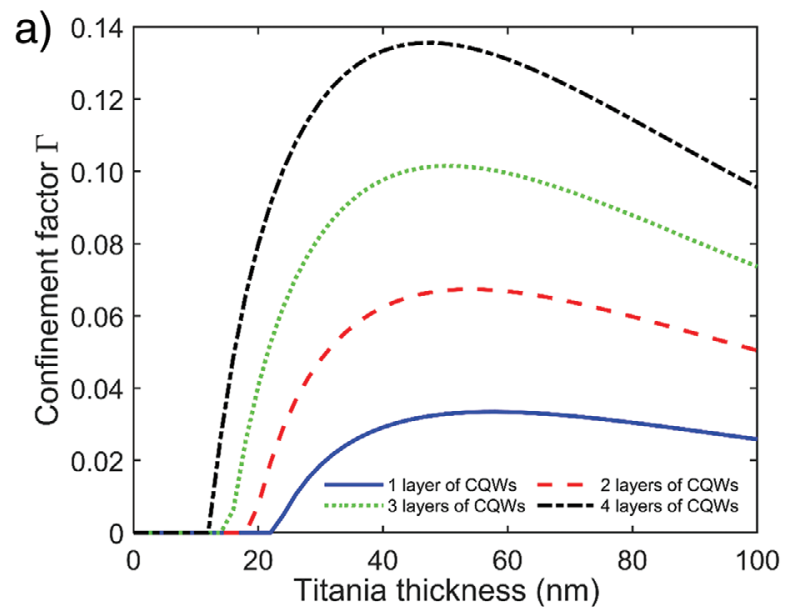

d)

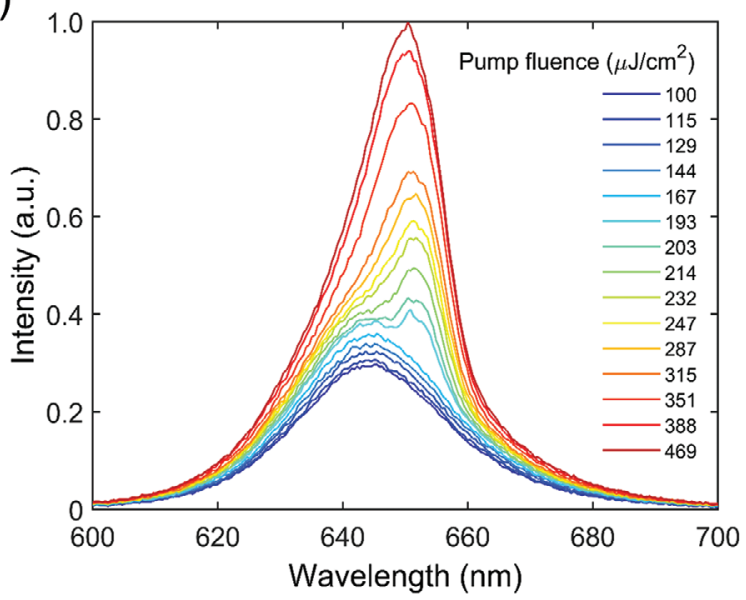

b)

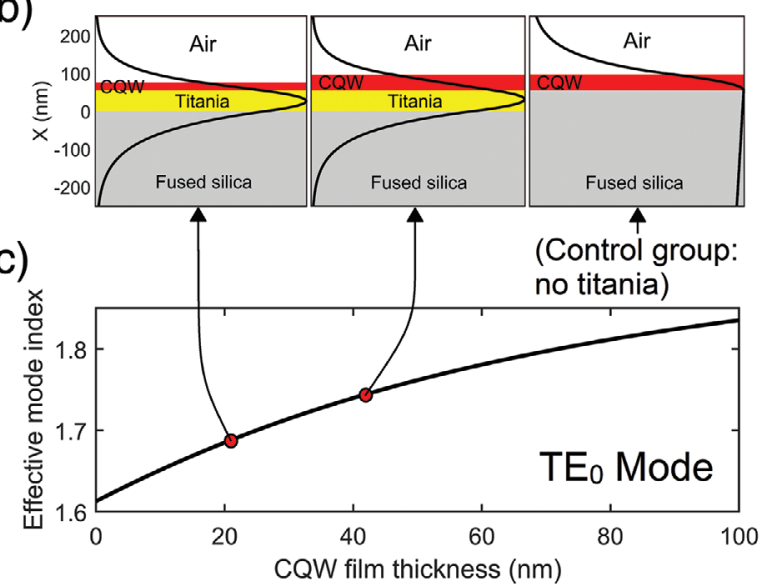

e)

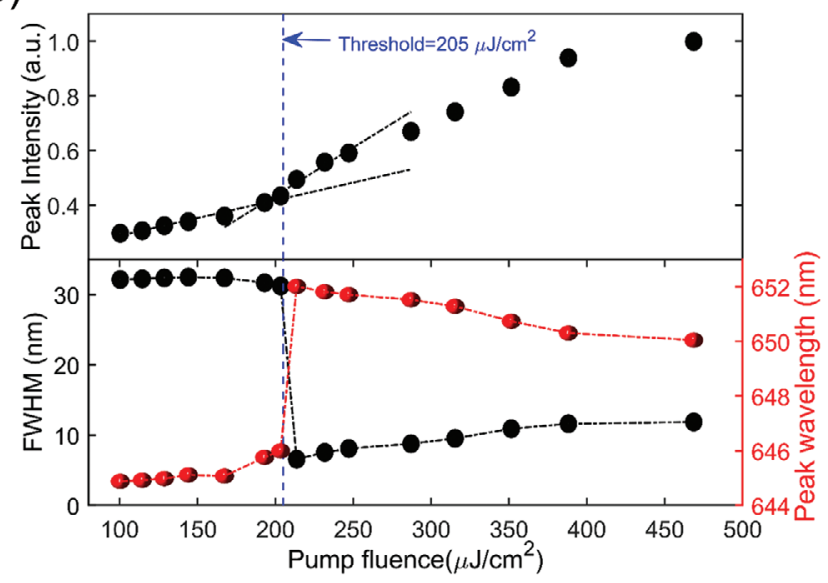

Figure 2. a) Confinement factor $\Gamma$ calculated as a function of $\mathrm{TiO}_{2}$ film thickness for 1 to 4 layers of $\mathrm{CQWs}$. b) Analysis of $\mathrm{TE}_{0}$ mode intensity profiles at $\lambda=650 \mathrm{~nm}$ across the device stack with 3 layers of CQWs on $55 \mathrm{~nm} \mathrm{TiO}{ }_{2}$ (left), 6 layers of CQWs on $55 \mathrm{~nm} \mathrm{TiO} 2$ (middle), and control group with 6 layers of CQWs on fused silica without $\mathrm{TiO}_{2}$ (right). c) Effective $\mathrm{TE}_{0}$ mode index of asymmetric waveguide as a function of CQW active film thickness. d) Emission spectra of the CQWs in asymmetric waveguide structure with $55 \mathrm{~nm} \mathrm{TiO}$ and 3 layers of CQWs under single-photon absorption excitation $\left(\lambda_{\text {exc }}=400 \mathrm{~nm}\right)$. e) Peak emission intensity, FWHM and peak emission wavelength as a function of the pump fluence, extracted from Gaussian fits for the spontaneous and ASE components.

the mode is more confined inside the $\mathrm{TiO}_{2}$ film and its share in the active CQW film reduces. The $\mathrm{TiO}_{2}$ thickness that would maximize the optical confinement across a layer of CQWs is calculated to be $55 \mathrm{~nm}$ via numerical simulations. For 2, 3, and $4 \mathrm{CQW}$ layers, the optimum thickness of $\mathrm{TiO}_{2}$ is still close to $55 \mathrm{~nm}$, albeit slightly thinner when using thicker CQW layers. Furthermore, it is seen that additional CQW layers provide a more effective optical confinement around the optimal $\mathrm{TiO}_{2}$ thickness.

The modal analysis of the asymmetric waveguide with a $55 \mathrm{~nm}$ thick $\mathrm{TiO}_{2}$ film, and three layers of CQWs is shown in Figure $2 \mathrm{~b}$ (left). The $\mathrm{TE}_{0}$ mode is confined in the waveguide with a $\Gamma$ of $10.2 \%$ inside the CQW film, and an effective mode index of 1.69 (Figure 2c). The mode confinement factor increases to $13.5 \%$ in the case of using four layers of CQWs. Since the net modal gain is given by $g_{\text {modal }}=\Gamma g_{\text {material }}-\alpha$, where $g_{\text {material }}$ is the material gain and $\alpha$ is the total modal loss, the enhancement of optical confinement should result in the enhancement of ASE because of the higher net gain. It is worth noting that without $\mathrm{TiO}_{2}$ film there is no mode supported in the CQW film below six layers. As shown in Figure 2b (middle), the mode is confined in a waveguide with six layers of CQWs on $55 \mathrm{~nm}$ thick $\mathrm{TiO}_{2}$, with a $\Gamma$ of $19.5 \%$. However, this mode is weakly guided for the case of six layers of CQWs on bare fused silica, with confinement of about $1 \%$, making it practically difficult to observe gain and requiring the highest quality CQW film (Figure 2b, right).

The PL spectra and ASE of the resulting asymmetric waveguide of CQWs were measured using the experimental setup described in the Experimental Section in order to investigate the ASE behavior. Using the asymmetric waveguide structure, the thinnest gain medium capable of ASE was three layers of CQWs, for which the PL spectra at different pump levels are shown in Figure 2d. The intensity of the emitted light grows linearly with the pump fluence but with a distinct change in slope at the pump threshold, as shown in Figure 2e. At low pump intensities the light emitted from the edge of the film exhibited a broad emission spectrum, with a full-width-athalf-maximum (FWHM) in the range of $30-35 \mathrm{~nm}$, which decreased to 7-12 nm at pump intensities above the threshold. 
As it is well known, the spectral narrowing in the emission spectrum and the change in the slope in the emission intensity with increasing pump fluence are signatures of the onset of ASE. ${ }^{[31]}$ The ASE peak shows a distinct red shift with respect to the spontaneous emission feature, which is the characteristic behavior of such type-I heterostructures. ${ }^{[32]}$ However, as previously demonstrated by our group, ${ }^{[17]}$ the amount of red-shift can be attributed to the variance of the mode confinement with propagation wavelength and its trade-off with the peak gain of the colloidal material.

After increasing the pump fluence up to a certain level, the ASE peak experiences a blue shift from its initial position, which needs further elaboration. This can be attributed to the reabsorption in the active film. Figure $1 \mathrm{~b}$ shows that the extinction coefficient of CQWs increases at shorter wavelengths. At lower pump fluences some portion of the active CQWs is not excited; as a result, the active film will have higher reabsorption at shorter wavelengths. Hence, the ASE peak, which has a FWHM of about $10 \mathrm{~nm}$, will build up at longer wavelengths. As the pump fluence increases, most of the CQWs are excited and reabsorption will not force any limitation anymore. As a result, the ASE peak blue shifts to some extent, since the waveguide has a higher confinement factor at shorter wavelengths.

To further increase the confinement factor in order to allow for more efficient ASE performance from ultra-thin CQW layers, we adapted another type of waveguide in which the CQW layer is sandwiched by $\mathrm{TiO}_{2}$ from both top and bottom (quasisymmetric waveguide). Unlike the asymmetric waveguide, for which the peak of the mode profile resides inside the $\mathrm{TiO}_{2}$ film, the symmetric structure allows to push the optical field distribution to peak inside the optically active layer, thereby providing a stronger mode confinement. To achieve this, we carefully designed the refractive index profile of quasi-symmetric waveguide comprised of CQW layers by tuning the thicknesses of the top and bottom $\mathrm{TiO}_{2}$ films. The optimal thicknesses of the top and bottom $\mathrm{TiO}_{2}$ layers were found to be 50 and $40 \mathrm{~nm}$, respectively.

Figure 3a shows the cross-sectional TEM image of fabricated waveguide structure embedding two CQW layers a)

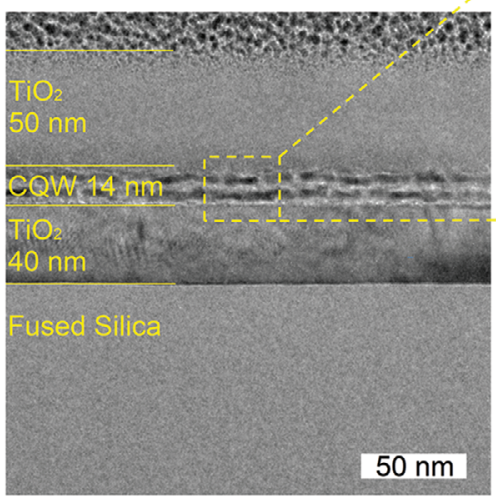

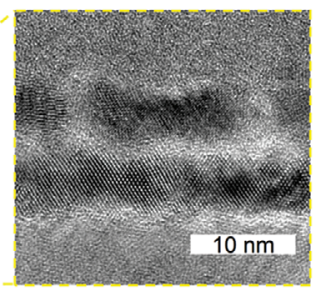

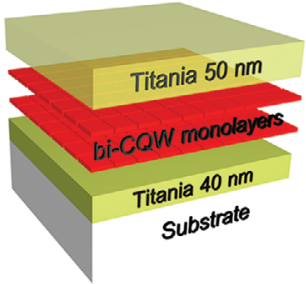

b)
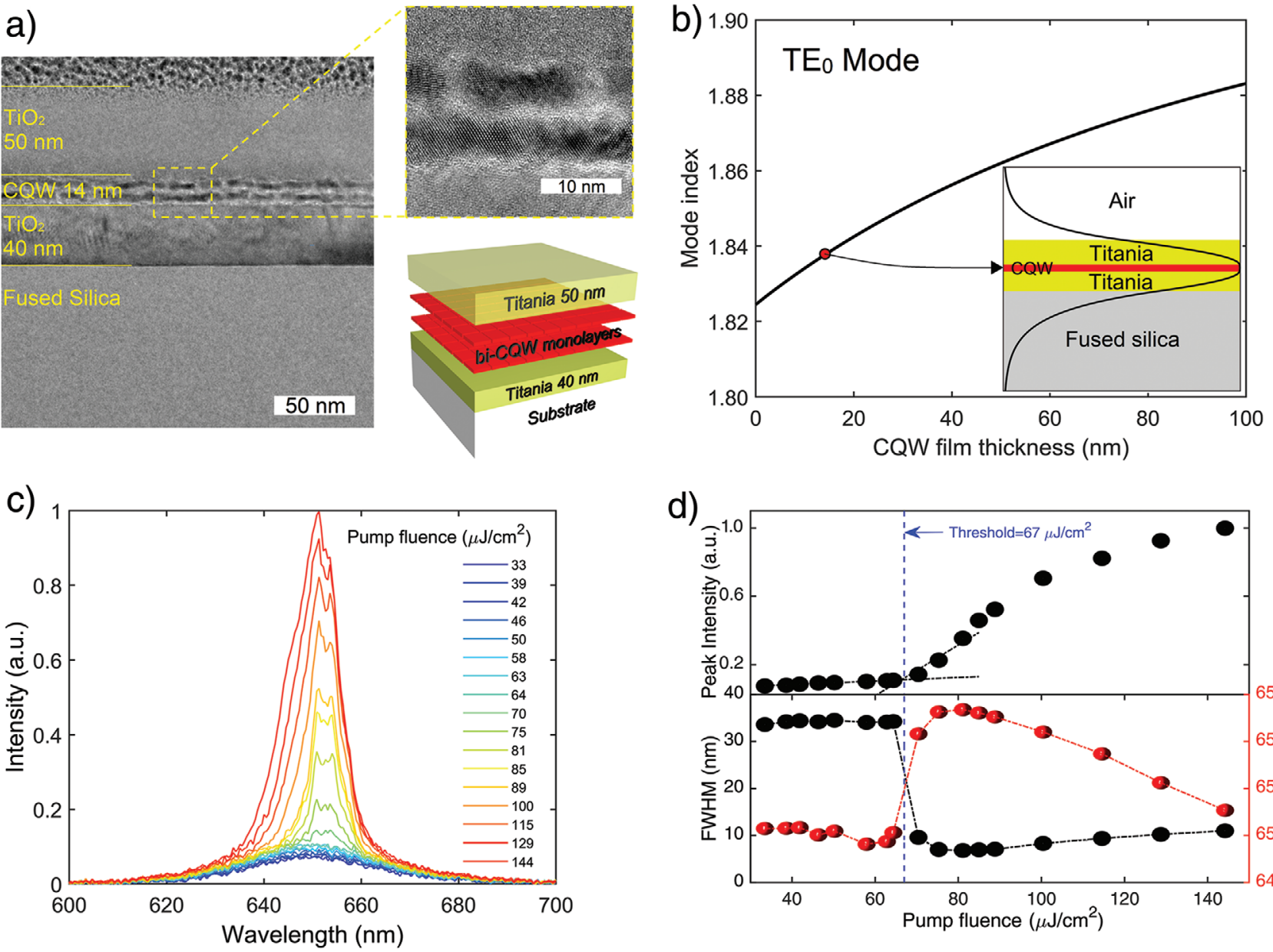

d)

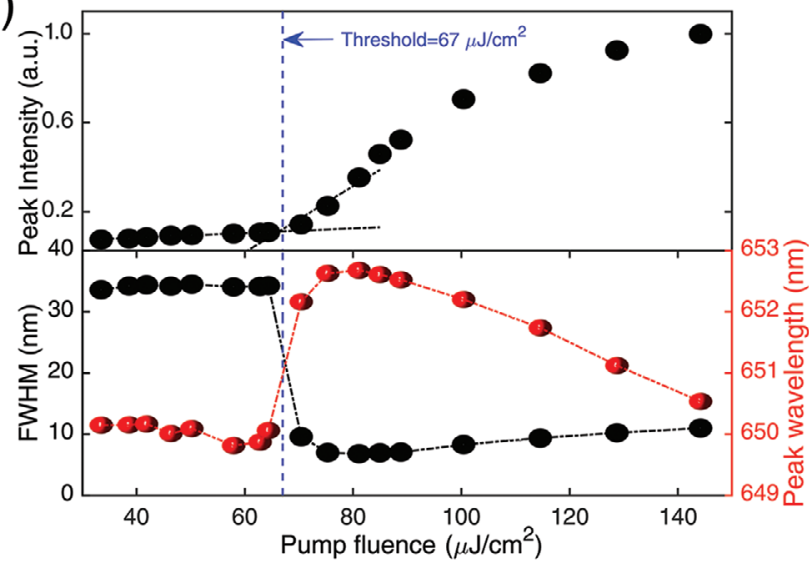

Figure 3. a) A cross-sectional TEM image of the quasi-symmetric waveguide (the scale bar is $50 \mathrm{~nm}$ ). The top layer is platinum deposited for protection in sample preparation process (fused ion beam) for imaging. b) Effective $\mathrm{TE}_{0}$ mode index of quasi-symmetric waveguide as a function of the $\mathrm{CQW}$ active film thickness. The wavelength is fixed at $650 \mathrm{~nm}$ and the top and bottom $\mathrm{TiO}_{2}$ film thicknesses are fixed at 50 and $40 \mathrm{~nm}$, respectively. c) Emission spectra of the CQWs in quasi-symmetric waveguide structure under single-photon excitation $\left(\lambda_{\text {exc }}=400 \mathrm{~nm}\right)$. d) Peak emission intensity, FWHM and peak emission wavelength as a function of the pump fluence, extracted from Gaussian fits for the spontaneous and ASE components spectra. 

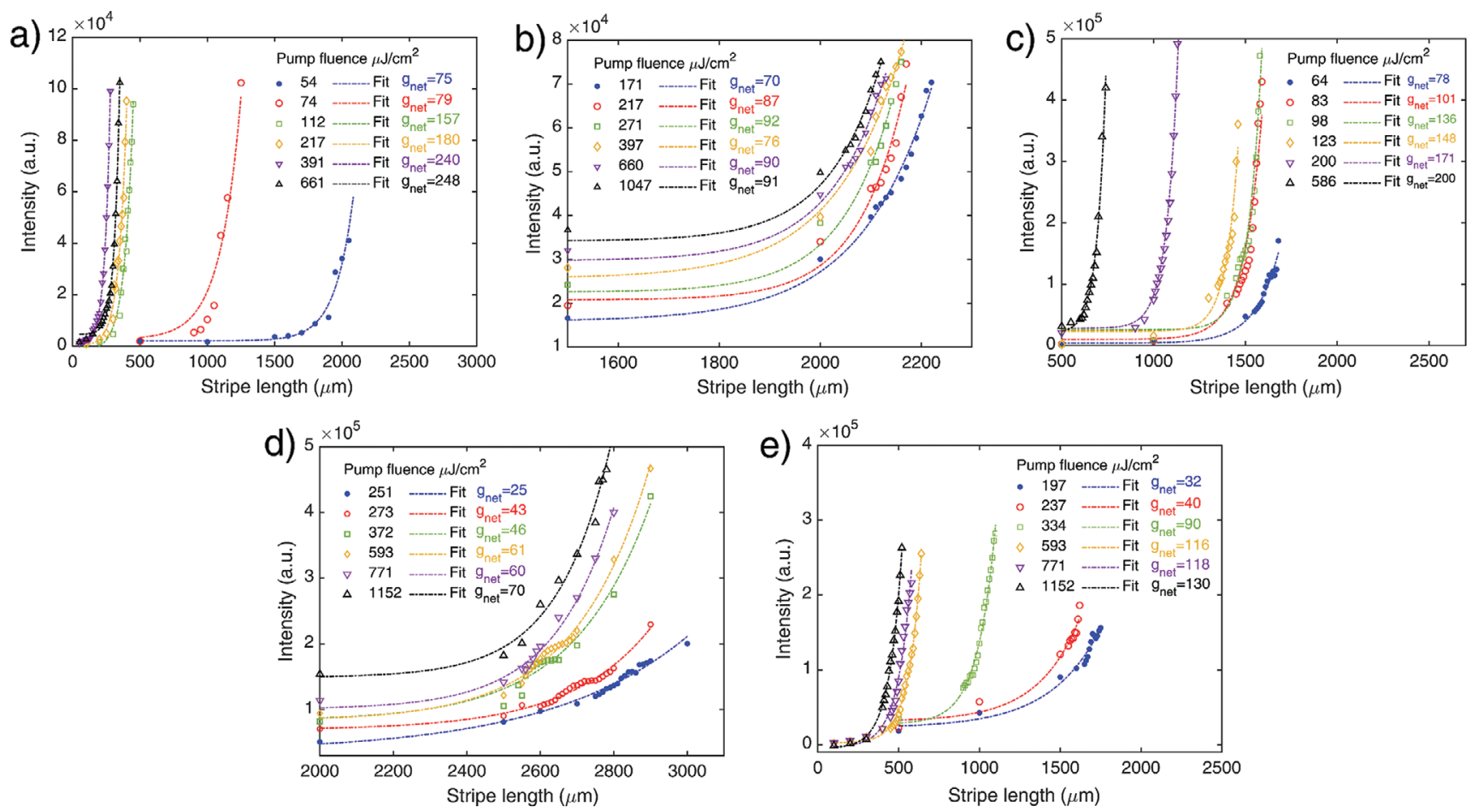

Figure 4. VSL measurements of the active waveguide structures parameterized with respect to the pumping fluence. Here, the dash-dotted lines show the fitting curves of the VSL data using Equation (2). a) 7 layers of CQWs on bare silica. b) Symmetric waveguide with 2 layers of CQWs. c) Symmetric waveguide with 3 layers of CQWs. d) Asymmetric waveguide with 3 layers of CQWs. e) Asymmetric waveguide with 4 layers of CQWs. All samples use the same CQWs.

sandwiched between 40 and $50 \mathrm{~nm} \mathrm{TiO}_{2}$ films. The enlarged image of CQWs in Figure 3a indicates that the CQWs and the capped ligands remain intact after being sandwiched between $\mathrm{TiO}_{2}$ layers. Colloidal nanocrystal films with oleic acid ligands are stable at temperatures up to $200{ }^{\circ} \mathrm{C}$ due to their minimal content of extra ligands. ${ }^{[25]}$ Figure 3 a also schematically depicts the overall waveguide structure. The CQWs synthesized by HIS technique exhibit good thermal stability at temperatures up to $200{ }^{\circ} \mathrm{C},[28]$ because of which their emission is fully preserved even after the high-temperature ALD process.

To analyze the influence of CQW film thickness, effective mode index of the $\mathrm{TE}_{0}$ mode as a function of the CQW thickness was computed, which is plotted in Figure 3b. Our calculations show that, if the wavelength is set to $650 \mathrm{~nm}$, with two layers of CQWs (14 nm thick), the quasi-symmetric waveguide can support the $\mathrm{TE}_{0}$ mode with an effective mode index of about 1.84. The normalized $\mathrm{TE}_{0}$ mode profile in the inset of Figure $3 \mathrm{~b}$ shows that the optical mode peak resides in the center of CQW film, which maximizes its confinement in the quasi-symmetric design. This confinement factor in the case of using bi-layers of CQWs is about $10.4 \%$, which shows a good improvement compared to the asymmetric waveguide (with a $\Gamma$ of about $7 \%$ ). Using this design we were able to observe ASE from two layers of CQWs for which the PL spectra at different pump levels are shown in Figure 3c. Distinct change in the slope at the pump threshold, spectral narrowing and red shift in the emission peak are all observed as indicators of ASE, shown in Figure 3d. The calculations of these guided modes yield consistent results with the ASE measurements in that the ASE is observed in our hybrid $\mathrm{TiO}_{2} / \mathrm{CQW}$ structures when the confinement factor is over $10 \%$.

To investigate the gain performance of these CQW waveguide structures, we measured $g_{\text {modal }}$ using variable stripe length (VSL) method, through which the intensity of emission was measured as a function of the excitation length under different pump fluences. ${ }^{[33]}$ Then $g_{\text {modal }}$ was calculated by fitting the experimental data to the following relation

$I(l, \lambda)=\frac{I_{\mathrm{sp}}(\lambda)}{g_{\operatorname{modal}(\lambda)}}\left(e^{g_{\text {modal }}(\lambda) . l}-1\right)$

where $I(l, \lambda)$ is the ASE intensity at the wavelength $\lambda, I_{\mathrm{sp}}(\lambda)$ is a constant proportional to the spontaneous emission intensity, and $l$ is the length of the pumped stripe in VSL measurements. The VSL measurement results for different types of waveguides with varied thicknesses are shown in Figure 4. Here, although different samples using either pure CQW monolayers on fused silica substrate (Figures 4a) or a hybrid waveguide structure, quasi-symmetric (Figure 4b,c) or asymmetric (Figure 4d,e), contain different number of CQW layers, they are all made up of the same CQWs.

For all waveguide structures, the extracted $g_{\text {modal }}$ is plotted as a function of the pump fluence in Figure 5a, and $g_{\text {modal }}$ values are fitted to the following equation

$g_{\text {modal }}=\Gamma g_{\text {material }}-\alpha=\Gamma \sigma_{\mathrm{e}} N_{\text {pop.inv. }}-\alpha$ 

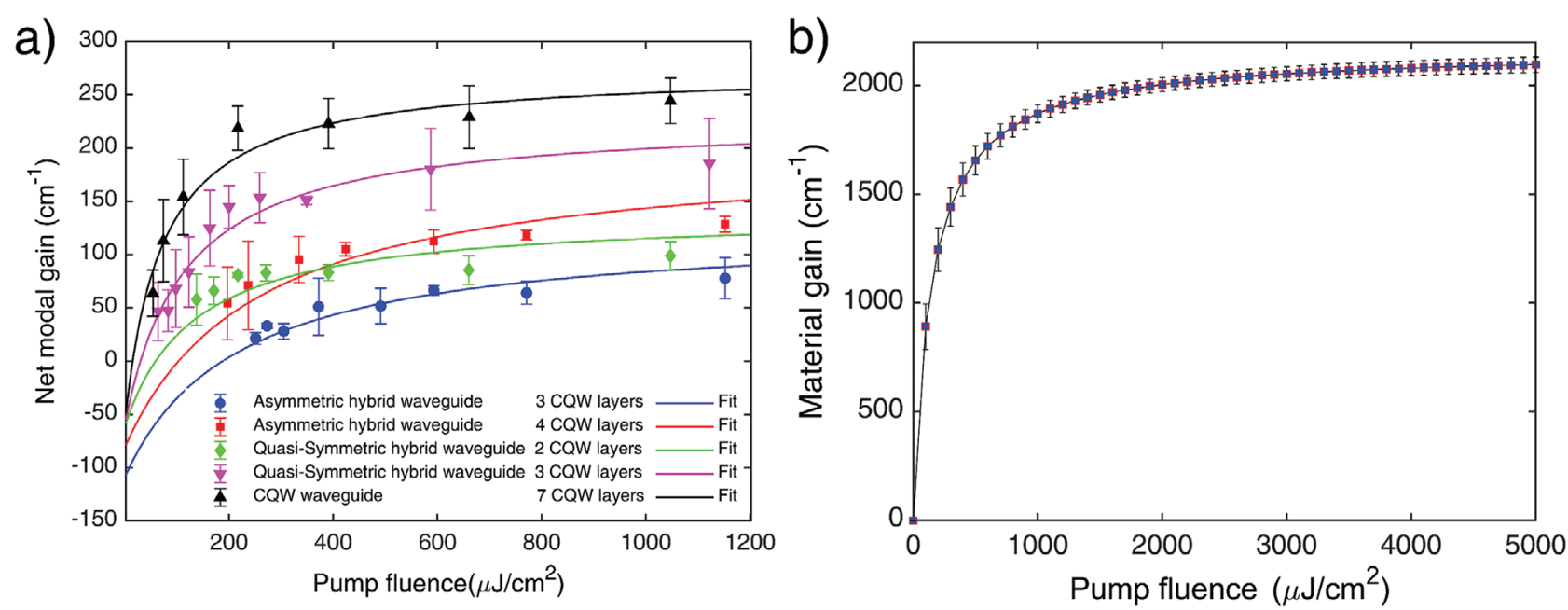

Figure 5. a) Pump-dependent net modal gain coefficients $g_{\text {modal }}$ for different types of waveguides (symbols). Each point in the graph is an average of three measurements indicated with their corresponding standard deviations. The solid line indicates the numerical fit to the experimental data using Equation (4). b) Material gain extracted from data fittings to Equation (4). The solid line is the material gain calculated using the extracted data from 5 different samples listed in Table 1.

$g_{\text {modal }}=\Gamma \sigma_{\mathrm{e}} n_{\mathrm{t}} \frac{\Phi_{\mathrm{p}} / \Phi_{\mathrm{s}}}{1+\Phi_{\mathrm{p}} / \Phi_{\mathrm{s}}}-\alpha$

where $\Gamma$ is the modal confinement calculated for each structure, $\sigma_{\mathrm{e}}$ is the stimulated emission cross-section, $N_{\text {pop.inv. }}$ is the density of the population inversion, $n_{\mathrm{t}}$ is the density of active centers, $\Phi_{\mathrm{p}}$ is the pump fluence, and $\Phi_{\mathrm{s}}$ is the saturation pump fluence. ${ }^{[34,35]}$ To further investigate the optical gain performance of our CdSe/Cd ${ }_{0.25} \mathrm{Zn}_{0.75} \mathrm{~S}$ core/HIS CQWs, we fitted the $g_{\text {modal }}$ data extracted from VSL measurements to Equation (4) to extract material gain parameters. The solid lines in Figure 5a are the best fits using Equation (4). The parameters producing those fits are listed in Table 1 for all structures.

According to Equation (4), at zero pump fluence we have $g_{\text {modal }}=-\alpha$, which means that using the fitted curve we can find the total losses of each waveguide. ASE threshold fluence can be defined as the pump fluence for which $g_{\text {modal }}=0$. The thresholds obtained from the fitted curves for each structure match well the thresholds deduced from the ASE measurements. The ASE thresholds as well as the loss coefficients are listed in Table 1 for all samples. The average number of excitons per CQW corresponding to optical gain threshold $(\mathrm{N})$ is calculated as 2.34 for the lowest fluence of $18 \mu \mathrm{J} \mathrm{cm}^{-2}$ at which we observed ASE (see the Supporting Information for calculation method).

The fitting results show that the CQW waveguide without $\mathrm{TiO}_{2}$ layer has the lowest $\Phi_{\mathrm{s}}$. This stems from the fact that 7-layer thick CQW active film guides $400 \mathrm{~nm}$ pump light effectively and, consequently, $\Phi_{\mathrm{s}}$ will reduce. However, in the quasi- symmetric and asymmetric waveguides, some portion of the pump is guided in $\mathrm{TiO}_{2}$ films, which thus reduces the pumping efficiency. Also, in the quasi-symmetric waveguide the pump field will be guided better in CQW film due to symmetry, hence the active medium will be pumped more efficiently compared to the asymmetric waveguide.

The 7-layer CQW waveguide experiences lower loss compared to the other waveguide structures, which is possibly as a result of the uniformity of this thick CQW structure. On the other hand, in other structures, we have multilayers of different materials deposited using different methods which can result in scattering losses especially at the boundaries. These numbers compare well to or are better than the loss coefficients previously reported in thicker films of colloidal semiconductor nanocrystals including those of QDs ${ }^{[36]}$ and CQWs, ${ }^{[20]}$ with film thickness of about hundreds of nanometers. We ascribe these low losses to the reduction in surface roughness and absence of random orientation of CQWs in our closely packed self-assembled films, resulting in decreased scattering.

The values in Table 1 show a small amount of variation in $\sigma_{\mathrm{e}}$, which needs more consideration. This parameter is a characteristic property of the CQWs and should be independent of the waveguide structure and the thickness of gain medium. The results indicate that $\sigma_{\mathrm{e}}$ reduces slightly by increasing the thickness of active medium in every waveguide design. The lowest value results from the 7-layer CQW waveguide. The reduction of $\sigma_{\mathrm{e}}$ is possibly due to one of the simplifying assumptions used in the derivations of Equation (4). In this relation it is assumed that the pumping is constant and homogeneous inside

Table 1. Parameters extracted from the net modal gain data analysis.

\begin{tabular}{lcccccc}
\hline Type of Waveguide & Number of CQW layers & CQW film Thickness $[\mathrm{nm}]$ & $\Phi_{\mathrm{th}}\left[\mu \mathrm{cm}^{-2}\right]$ & $\Phi_{\mathrm{s}}\left[\mu \mathrm{cm}^{-2}\right]$ & $\alpha\left[\mathrm{cm}^{-1}\right]$ & $\sigma_{\mathrm{e}} \times 10^{-15}\left[\mathrm{~cm}^{2}\right]$ \\
\hline Asymmetric hybrid & 3 & 21 & 205 & 216 & 109 & 4.78 \\
& 4 & 28 & 112 & 256 & 81 & 4.51 \\
Quasi-symmetric hybrid & 2 & 14 & 67 & 133 & 61 & 4.63 \\
& 3 & 21 & 49 & 115 & 70 & 58 \\
CQW pure & 7 & 49 & 18 & 71 & 4.30 \\
\hline
\end{tabular}


the whole active medium. However, in reality, the pumping is both spatially and temporally inhomogeneous. This results in a variation of the population inversion with depth and in time. As a result, the experimentally measured gain is a mean value resulting from the temporal and spatial averaging of the population inversion in different samples. Therefore, in Equation (4), which does not consider the pump inhomogeneity, $N_{\text {pop.inv. }}$ is assumed to be constant throughout the active medium. Hence, by fitting experimental results such as those fitted to Equation (4) and plotted in Figure 5a, the inhomogeneity translates to the slight variation in $\sigma_{\mathrm{e}}$. This means that the $\sigma_{\mathrm{e}}$ levels listed in Table 1 should be considered as effective values.

Because of the aforementioned reasoning the calculated material gain from the fitting parameters related to each sample yields slightly different results. The average of these results from 5 samples is plotted in Figure $5 \mathrm{~b}$ together with the standard error of the mean. The calculations yield $g_{\text {material }}$ to be as large as $2100 \pm 100 \mathrm{~cm}^{-1}$ at $5000 \mu \mathrm{J} \mathrm{cm}^{-2}$ pump fluence, with an upper limit of about $2300 \mathrm{~cm}^{-1}$. The values obtained using this methodology can give comparative results on the quality of the gain medium.

These attained net modal gains are from the thinnest gain medium among all colloidal materials reported so far, to the best of our knowledge. It is worth mentioning that here we have achieved the gain from the thin active medium inside a waveguide without any resonance behavior of an optical cavity. Roh et al. ${ }^{[37]}$ obtained gain with a threshold of $18.8 \mu \mathrm{J} \mathrm{cm}^{-2}$ from a QD gain medium as thin as $50 \mathrm{~nm}$ using a distributed feedback (DFB) resonator design. The propagation of the excitation and emission light along the polymeric waveguide with integrated multilayers of $\mathrm{CdSe} / \mathrm{CdS} / \mathrm{ZnCdS}$ core/crown/shell CQWs have been studied by Suarez et al. ${ }^{[26]}$ However, ASE from their multilayer structure was not possible due to the low modal confinement in such a waveguide. Integration of dye gain media into optical waveguides have also been reported in previous studies. Kretsch et al. obtained a net modal gain of $g_{\text {modal }}=15-20 \mathrm{~cm}^{-1}$ at a pumping intensity of $0.87 \mathrm{MW} \mathrm{cm}^{-2}$ at $355 \mathrm{~nm}$ wavelength using stilbenoid and perylenevinylene derivative in polystyrene waveguides. ${ }^{[38]}$ Yuyama et al. obtained a net gain of $14 \mathrm{~cm}^{-1}$ from a $9.3 \mu \mathrm{m}$ thick planar waveguide of fluorinated-polyimide doped with LDS950 dye with a pumping intensity of $0.74 \mathrm{~mJ} \mathrm{~cm}{ }^{-2}$ at $337 \mathrm{~nm}$ wavelength. ${ }^{[39]}$ From the above comparisons, it is seen that the values of the gain threshold and the net gain levels achieved in this work compare well with or prove better than those reported in the literature. Here, the side-by-side assembly of CQWs in their solid films, all of them being oriented face-down coplanar with the waveguide structures, makes the resulting close-packed CQW films to exhibit a relatively high refractive index and low scattering losses, and integrating them in carefully designed multilayered hybrid waveguides increases optical confinement factors.

\section{Conclusions}

In conclusion, we demonstrated record thin colloidal gain medium from bi-CQW layers, enabled by the integration and mode confinement of high index dielectrics and close-packed self-assembled layers of $\mathrm{CdSe} / \mathrm{Cd}_{0.25} \mathrm{Zn}_{0.75} \mathrm{~S}$ core/HIS CQWs.
Carefully designed refractive index profile across a quasi-symmetric waveguide structure using the high packing density of the self-assembled CQW films, combined with the exploitation of the HIS technique, rendering the CQWs with a near-unity PL quantum yield, reduced nonradiative recombination, smooth film surface, and enhanced stability, together enable gain from such an ultra-thin CQW film. We demonstrate that the proposed hybrid waveguide structure represents a robust gain platform, which is tolerant of variation of device structural parameters including thickness of CQW layers, exhibits stable ASE behavior over time and is capable of low-threshold ASE even with ultrathin $\approx 14 \mathrm{~nm}$ thick bi-CQW layers. Considering the realization of optical gain from an ultra-thin CQWs film, simple fabrication technique and spectral tunability, this hybrid waveguide architecture can be applied to make on-chip active photonic colloidal devices for a wide range of applications including biophotonics and biosensing, and may enable electrically driven CQW-lasers.

\section{Experimental Section}

Synthesis of CdSe Core CQWs: $4 \mathrm{ML}$ CdSe core CQWs were synthesized according to the previously reported studies. ${ }^{[28]}$ Preparation of the cadmium myristate precursor: Sodium myristate and cadmium nitrate tetrahydrate both were separately dissolved in methanol and then they were mixed in a bottle by strong agitation until the mixture became bulky. Thereafter, the solution was filtered and washed three times with methanol and finally dried under vacuum for a day. $4 \mathrm{ML}$ thick $\mathrm{CdSe}$ core synthesis: $30 \mathrm{~mL}$ of octadecene, $20 \mathrm{mg}$ of Se, and $340 \mathrm{mg}$ of $\mathrm{Cd}$ myristate were reacted in a $100 \mathrm{~mL}$ three-neck flask and kept at $95{ }^{\circ} \mathrm{C}$ under vacuum for an hour. Then, the flask was heated to $240{ }^{\circ} \mathrm{C}$ under argon-gas flow and $100 \mathrm{mg}$ of cadmium acetate dihydrate was quickly added into the reaction flask at $195{ }^{\circ} \mathrm{C}$. The solution was kept for $10 \mathrm{~min}$ at $240{ }^{\circ} \mathrm{C}$ and the reaction flask was cooled to room temperature (RT) with water-bath after $1 \mathrm{~mL}$ of oleic acid injection. $6 \mathrm{~mL}$ of hexane was added into the solution at RT and precipitation was carried out by ethanol using the centrifugation at $6000 \mathrm{rpm}$ for $5 \mathrm{~min}$. Precipitated core CQWs were dissolved in hexane.

Synthesis of $\mathrm{CdSe} / \mathrm{Cd}_{0.25} Z_{0} n_{0.75} \mathrm{~S}$ Core/HIS CQWs: The synthesis method and recipe of the core/alloyed HIS CQWs are nearly similar to the previous reports. ${ }^{[9,10]} 4 \mathrm{ML}$ thick CdSe core CQWs solution in hexane with an optical density of 2 at $350 \mathrm{~nm}$ wavelength (using $1 \mathrm{~cm}$ long optical path) was used. $55.04 \mathrm{mg} \mathrm{Zn}$-acetate, $5 \mathrm{~mL}$ of 1-octadecene (ODE), $23.06 \mathrm{mg}$ of $\mathrm{Cd}$-acetate, $0.70 \mathrm{~mL}$ oleic acid, and $1 \mathrm{~mL}$ of CdSe core were mixed in a three-necked quartz flask $(50 \mathrm{~mL})$. The solution was kept under vacuum for $75 \mathrm{~min}$ at room temperature and thereafter for $45 \mathrm{~min}$ at $90{ }^{\circ} \mathrm{C}$ to remove impurities. After injection of $0.70 \mathrm{~mL}$ of oleylamine at $90{ }^{\circ} \mathrm{C}$ under argon-gas flow, the temperature was raised to $300^{\circ} \mathrm{C} .140 \mu \mathrm{L}$ of 1-octanethiol as $S$ source with $5 \mathrm{~mL}$ of ODE were mixed inside the glovebox and, as the temperature reached to $162^{\circ} \mathrm{C}$, the injection of this mixture was started at the rate of $10 \mathrm{~mL} \mathrm{~h}^{-1}$ until $240{ }^{\circ} \mathrm{C}$ and then the rate was reduced to $5 \mathrm{~mL} \mathrm{~h}^{-1}$ until all the precursor was injected. The solution was kept at $300{ }^{\circ} \mathrm{C}$ for an hour and then cooled to room temperature in water-bath. $5 \mathrm{~mL}$ of hexane was added to the quenched mixture. The solution was precipitated with $8 \mathrm{~mL}$ of ethanol by centrifuging at $6000 \mathrm{rpm}$ for $5 \mathrm{~min}$, and the precipitate was redispersed in $4 \mathrm{~mL}$ of hexane. The sample was cleaned with ethanol two times more before usage.

Sample Characterization: $\mathrm{PL}$ and absorption characterization of the samples were carried out by using Cary Eclipse Fluorescence Spectrophotometer and UV-visible spectrophotometer of Varian Cary 100 instruments. Structural characterizations were performed by exploiting TEM images using energy-dispersive $X$-ray spectroscopy with Tecnai G2 $\mathrm{F} 30$ at $300 \mathrm{kV}$. Quantum yield (QY) of the samples was measured by using absolute QY measurements system and the methodology defined by de Mello et al. ${ }^{[40]}$ 
Waveguide Modal Analysis: The modal analyses were carried out using a commercially available software package (Lumerical MODE solution). 2D simulations were performed using perfectly matched layer $(P M L)$ absorbing boundary condition $(A B C)$ in a direction normal to the waveguide surface and periodic boundary condition in lateral direction. The experimental refractive indices of the fused silica, $\mathrm{TiO}_{2}$ and $\mathrm{CQW}$ films were extracted using ellipsometric measurements.

Optical Measurements: As the pump excitation, a femtosecond mode-locked Ti: sapphire amplifier (Spectra Physics, Spitfire Pro) was used with a $1 \mathrm{kHz}$ repetition rate and a $120 \mathrm{fs}$ pulse width, operating at $800 \mathrm{~nm}$ wavelength, which is frequency-doubled with a BBO crystal at the output to $400 \mathrm{~nm}$. The pump fluence on the sample was changed using a variable neutral density filter. A cylindrical lens with a $15 \mathrm{~cm}$ focal length was employed for the stripe excitation. The PL signal was collected in the direction perpendicular to the excitation by means of a fiber coupled to a spectrometer (Maya 2000 Pro). The VSL measurement method was used to evaluate the net gain of the samples, which consists of optically pumping the sample with a beam of variable stripe length at constant fluence. For VSL measurements, the optical setup was slightly changed and an adjustable slit was placed right before the sample to vary the length of excitation stripe. To minimize the light diffraction effects, the sample was placed at the vicinity of this slit $(\approx 4-5 \mathrm{~mm})$.

Atomic Layer Deposition (ALD) of $\mathrm{TiO}_{2}$ Thin Film: All $\mathrm{TiO}_{2}$ layers were deposited in Savannah; Cambridge Nanotech ALD reactor with $\mathrm{Ti}\left(\mathrm{NMe}_{2}\right)_{4}$ and $\mathrm{H}_{2} \mathrm{O}$ as alternating precursors of $\mathrm{Ti}$ and $\mathrm{O}$, respectively. In each ALD reaction cycle, the reaction chamber was replenished with precursors by a $0.15 \mathrm{~s}$ pulse and then purged with $\mathrm{N}_{2}$ for $20 \mathrm{~s}$. The deposition temperature were kept at $150^{\circ} \mathrm{C}$. The film growth per cycle was found to be around $0.4 \mathrm{~A}$ per cycle without significant variation.

Self-Assembly of CQW Monolayers: The previously reported recipe for the self-assembly of $\mathrm{CdSe} / \mathrm{CdZnS} \mathrm{CQW}$ s with some modifications was used:[17] Substrates predeposited with $\mathrm{TiO}_{2}$ were fixed onto a slightly tilted stage by $10^{\circ}$ and immersed into diethylene glycol (DEG) that is contained in a teflon dish. CQW solution $\left(10 \mathrm{mg} \mathrm{mL}^{-1}\right)$ in hexane was dropped in an amount sufficient to cover most of the DEG interface. After the evaporation of the CQW solution, silicone oil (silicone elastomer, Sylgard 184) dissolved in hexane $\left(2 \mathrm{mg} \mathrm{mL}^{-1}\right)$ was dropped from one edge of the DEG interface to compress the CQW membrane. DEG was drained out with a peristaltic pump at a rate of $\left(40 \mu \mathrm{L} \mathrm{min}^{-1}\right)$. Residual DEG on substrates was dried under vacuum at room temperature. For multilayered deposition, this process was repeated onto previously deposited substrates as many times as needed.

Scanning Electron Microscopy (SEM) / Transmission Electron Microscopy (TEM): All SEM images were acquired using a FEI NovaLab 600i SEM/ FIB instrument. FIB (focused ion beam) milling was used to prepare debris free thin film of sandwiched $\mathrm{CQW}$ layers between $\mathrm{TiO}_{2}$ layers for TEM cross-sectional inspections. The area of interest was protected with deposition of a thin carbon layer and platinum before etching. The TEM analysis was performed using JEOL $2100 \mathrm{~F}$ electron microscope.

Ellipsometry: The thickness and optical parameters of CQW and $\mathrm{TiO}_{2}$ films were determined using J.A.Woollam Co., Inc variable angle spectroscopic ellipsometer (VASE). For $\mathrm{TiO}_{2}$ the changes in ellipsometric $\Psi$ and $\Delta$ data were measured at three angles of incidence $\left(55^{\circ}, 60^{\circ}\right.$, and $65^{\circ}$ ) over the spectral range of $400-700 \mathrm{~nm}$ in steps of $10 \mathrm{~nm}$ and subsequently used to extract the refractive index $(n)$ and the extinction coefficient $(k)$ of films. The complex refractive index of the thin CQW films was determined using ellipsometry in accordance with the previous literature. ${ }^{[47,42]}$ Ellipsometric data was collected from a multilayered CQW sample deposited on silicon at $400-1000 \mathrm{~nm}$ range with $10 \mathrm{~nm}$ steps and at three different incidence angles of $65^{\circ}, 70^{\circ}$, and $75^{\circ}$. The thickness of the sample was determined from the spectral region across which the CQWs are non-absorbing $(700-1000 \mathrm{~nm})$ using a Cauchy model. ${ }^{[43]}$ This thickness was kept fixed and the wavelength range was gradually expanded to fit the excitonic features using Kramers-König consistent dispersion curves. The complex dielectric function was decomposed into one Tauc-Lorentz oscillator and three Gaussian oscillators. The acquired data analysis was performed using W-Vase32 software package.

\section{Supporting Information}

Supporting Information is available from the Wiley Online Library or from the author. Description of CQW concentration effect on surface coverage of the self-assembly, calculation of effective refractive index of the self-assembled films, measured refractive index of $\mathrm{TiO}_{2}, \mathrm{ASE}$ measurements of other samples, calculation of average number of excitons, ASE operation time measurement.

\section{Acknowledgements}

The authors acknowledge the financial support in part from NRFNRFI2016-08 and in part from TUBITAK 115 F279 and 117E713. H.V.D. gratefully acknowledges support from TUBA. O.E. acknowledges TUBITAK for the financial support through BIDEB 2211 program. The authors thank Mr. Mustafa Guler for TEM imaging of the as-synthesized CQWs and preparation of the TEM cross-sectional sample and Dr. Gokce Celik for her help on the ellipsometric measurements.

\section{Conflict of Interest}

The authors declare no conflict of interest.

\section{Keywords}

colloidal quantum wells, liquid-air interface self-assembly, optical gain, optical mode confinement, ultra-thin waveguides

Received: July 16, 2020

Revised: August 26, 2020

Published online: October 20, 2020

[1] J. Zhao, Y. Yan, Z. Gao, Y. Du, H. Dong, J. Yao, Y. S. Zhao, Nat. Commun. 2019, 10, 870.

[2] Y. Jia, R. A. Kerner, A. J. Grede, B. P. Rand, N. C. Giebink, Nat. Photonics 2017, 11, 784.

[3] V. I. Klimov, A. A. Mikhailovsky, S. Xu, A. Malko, J. A. Hollingsworth, C. A. Leatherdale, H. J. Eisler, M. G. Bawendi, Science 2000, $290,314$.

[4] B. T. Diroll, D. V. Talapin, R. D. Schaller, ACS Photonics 2017, 4, 576.

[5] B. Guzelturk, Y. Kelestemur, M. Olutas, S. Delikanli, H. V. Demir, ACS Nano 2014, 8, 6599.

[6] A. Rose, Z. Zhu, C. F. Madigan, T. M. Swager, V. Bulović, Nature 2005, 434, 876

[7] F. Vollmer, S. Arnold, Nat. Methods 2008, 5, 591.

[8] C. Vannahme, S. Klinkhammer, U. Lemmer, T. Mappes, Opt. Express 2011, 19, 8179 .

[9] Y. Altintas, K. Gungor, Y. Gao, M. Sak, U. Quliyeva, G. Bappi, E. Mutlugun, E. H. Sargent, H. V. Demir, ACS Nano 2019, 13, 10662.

[10] B. Liu, Y. Altintas, L. Wang, S. Shendre, M. Sharma, H. Sun, E. Mutlugun, H. V. Demir, Adv. Mater. 2020, 32, 1905824.

[11] A. A. Rossinelli, H. Rojo, A. S. Mule, M. Aellen, A. Cocina, E. De Leo, R. Schäublin, D. J. Norris, Chem. Mater. 2019, 31, 9567.

[12] Y. Kelestemur, Y. Shynkarenko, M. Anni, S. Yakunin, M. L. De Giorgi, M. V. Kovalenko, ACS Nano 2019, 13, 13899.

[13] M. D. Tessier, C. Javaux, I. Maksimovic, V. Loriette, B. Dubertret, ACS Nano 2012, 6, 6751.

[14] M. Sak, N. Taghipour, S. Delikanli, S. Shendre, I. Tanriover, S. Foroutan, Y. Gao, J. Yu, Z. Yanyan, S. Yoo, C. Dang, H. V. Demir, Adv. Funct. Mater. 2020, 30, 1907417.

[15] N. Taghipour, S. Delikanli, S. Shendre, M. Sak, M. Li, F. Isik, I. Tanriover, B. Guzelturk, T. C. Sum, H. V. Demir, Nat. Commun. 2020, 11, 3305. 
[16] C. She, I. Fedin, D. S. Dolzhnikov, P. D. Dahlberg, G. S. Engel, R. D. Schaller, D. V. Talapin, ACS Nano 2015, 9, 9475.

[17] O. Erdem, S. Foroutan, N. Gheshlaghi, B. Guzelturk, Y. Altintas, H. V. Demir, Nano Lett. 2020, 20, 6459.

[18] E. Baghani, S. K. Oleary, I. Fedin, D. V. Talapin, M. Pelton, J. Phys. Chem. Lett. 2015, 6, 1032.

[19] R. Vaxenburg, A. Rodina, E. Lifshitz, A. L. Efros, Nano Lett. 2016, 16, 2503.

[20] D. Dede, N. Taghipour, U. Quliyeva, M. Sak, Y. Kelestemur, K. Gungor, H. V. Demir, Chem. Mater. 2019, 31, 1818.

[21] J. Q. Grim, S. Christodoulou, F. Di Stasio, R. Krahne, R. Cingolani, L. Manna, I. Moreels, Nat. Nanotechnol. 2014, 9, 891.

[22] O. Erdem, K. Gungor, B. Guzelturk, I. Tanriover, M. Sak, M. Olutas, D. Dede, Y. Kelestemur, H. V. Demir, Nano Lett. 2019, 19, 4297.

[23] Y. Gao, M. C. Weidman, W. A. Tisdale, Nano Lett. 2017, 17, 3837.

[24] C. Dang, J. Lee, C. Breen, J. S. Steckel, S. Coe-Sullivan, A. Nurmikko, Nat. Nanotechnol. 2012, 7, 335.

[25] H. Kim, K. S. Cho, H. Jeong, J. Kim, C. W. Lee, W. K. Koh, Y. G. Roh, S. W. Hwang, Y. Park, ACS Photonics 2016, 3, 1536.

[26] I. Suarez, R. Munoz, V. Chirvony, J. P. Martinez-Pastor, M. Artemyev, A. Prudnikau, A. Antanovich, A. Mikhailov, IEEE J. Sel. Top. Quantum Electron. 2017, 23, 1.

[27] J. M. Winkler, F. T. Rabouw, A. A. Rossinelli, S. V. Jayanti, K. M. McPeak, D. K. Kim, B. Le Feber, F. Prins, D. J. Norris, Nano Lett. 2019, 19, 108.

[28] Y. Altintas, U. Quliyeva, K. Gungor, O. Erdem, Y. Kelestemur, E. Mutlugun, M. V. Kovalenko, H. V. Demir, Small 2019, 15, 1804854
[29] F. Feng, L. T. Nguyen, M. Nasilowski, B. Nadal, B. Dubertret, L. Coolen, A. Maître, Nano Res. 2018, 11, 3593.

[30] S. M. Lee, D. G. Cahill, T. H. Allen, Phys. Rev. B 1995, 52, 253.

[31] M. D. McGehee, R. Gupta, S. Veenstra, E. K. Miller, M. A. Díaz-García, A. J. Heeger, Phys. Rev. B: Condens. Matter Mater. Phys. 1998, 58, 7035.

[32] J. M. Pietryga, Y. S. Park, J. Lim, A. F. Fidler, W. K. Bae, S. Brovelli, V. I. Klimov, Chem. Rev. 2016, 116, 10513.

[33] K. L. Shaklee, R. E. Nahory, R. F. Leheny, J. Lumin. 1973, 7, 284.

[34] W. Koechner, Solid-State Laser Engineering, Springer-Verlag, New York 2013.

[35] B. Guzelturk, Y. Kelestemur, M. Olutas, Q. Li, T. Lian, H. V. Demir, J. Phys. Chem. Lett. 2017, 8, 5317.

[36] C. H. Lin, E. Lafalce, J. Jung, M. J. Smith, S. T. Malak, S. Aryal, Y. J. Yoon, Y. Zhai, Z. Lin, Z. V. Vardeny, V. V. Tsukruk, ACS Photonics 2016, 3, 647 .

[37] J. Roh, Y. S. Park, J. Lim, V. I. Klimov, Nat. Commun. 2020, 11, 271.

[38] K. P. Kretsch, C. Belton, S. Lipson, W. J. Blau, F. Z. Henari, H. Rost, S. Pfeiffer, A. Teuschel, H. Tillmann, H. H. Hörhold, J. Appl. Phys. 1999, 86, 6155.

[39] S. Yuyama, T. Nakajima, K. Yamashita, K. Oe, Appl. Phys. Lett. 2008, 93, 023306

[40] J. C. de Mello, H. F. Wittmann, R. H. Friend, Adv. Mater. 1997, 9, 230.

[41] B. T. Diroll, E. A. Gaulding, C. R. Kagan, C. B. Murray, Chem. Mater. 2015, 27, 6463.

[42] D. B. Dement, M. Puri, V. E. Ferry, J. Phys. Chem. C 2018, 122, 21557.

[43] W. A. McGahan, B. Johs, J. A. Woollam, Thin Solid Films 1993, 234, 443. 